\title{
Propagation of guided modes in curved nanoribbon waveguides
}

\author{
Zhuo Ye, ${ }^{\text {a) }}$ Xinhua Hu, Ming $\mathrm{Li}$, and Kai-Ming $\mathrm{Ho}^{\text {bi }}$
}

Ames Laboratory, Lowa State University, Ames, Lowa 50010 and Department of Physics and Astronomy,

lowa State University, Ames, lowa 50010

\section{Peidong Yang}

Department of Chemistry, University of California, Berkeley, California 94720 and Materials Science

Division, Lawrence Berkeley National Laboratory, I Cyclotron Road. Berkeley, California 94720

(Received 23 August 2006; accepted 30 October 2006; published online 12 December 2006)

The authors develop a plane-wave-based transfer matrix method in curvilinear coordinates to study the guided modes in curved nanoribbon waveguides. The problem of a curved structure is transformed into an equivalent one of a straight structure with spatially dependent tensors of dielectric constant and magnetic permeability. The authors investigate the coupling between the eigenmodes of the straight part and those of the curved part when the waveguide is bent.

Semiconductor nanowires and nanoribbons have many promising optoelectronic applications such as waveguides, ${ }^{1 / 2}$ lasers, ${ }^{3-5}$ optical switches, and sensors. ${ }^{6}$ Nanowaveguides are especially important as links between optoelectronic elements. While straight waveguides have been widely studied, it is much more complicated to study a waveguide and its eigenmode properties when it is bent into various shapes. The guided modes in a bent waveguide present different propagation properties from a straight one. In a recent work, Law et al. found strong regular oscillations in the output spectrum when light is traveling in a curved nanoribbon waveguide. The largest amplitude of oscillations is about $50 \%$ of the maximum output intensity.

The plane-wave-based transfer matrix method ${ }^{7,8}$ (TMM) is a powerful analytical tool to understand straight waveguides." However, current TMM is for Euclidian coordinate $(x, y, z)$ system only and cannot deal with curved waveguides. In this letter, we develop a new TMM in curvilinear coordinate system to study curved nanoribbon waveguides. We show that curved sections in waveguides can result in strong regular oscillations in the transmission spectrum similar to those in Ref. 1.

We start by considering an arc structure characterized by the radius of curvature $R$ and span length $s$ [as shown in Figs. 1(a) and 1(b)]. A bent nanoribbon waveguide of any general shape can be approximated by a series of arcs with different curvatures and spans. Following a previous work, ${ }^{10}$ we introduce curvilinear coordinates $\left(x^{\prime}, y^{\prime}, s\right)$ [Fig. l(b)] and in this coordinate system Maxwell's equations can be written in terms of differential equations relating to the transverse components of fields:

$$
\begin{aligned}
& \frac{\partial}{\partial s} E_{x^{\prime}}=\frac{1}{-i k_{0}} \frac{\partial}{\partial x^{\prime}}\left[\frac{1}{\varepsilon_{3}}\left(\frac{\partial H_{y^{\prime}}}{\partial x^{\prime}}-\frac{\partial H_{x^{\prime}}}{\partial y^{\prime}}\right)\right]+i k_{0} \mu_{2} H_{y^{\prime}}, \\
& \frac{\partial}{\partial s} E_{y^{\prime}}=\frac{1}{-i k_{0}} \frac{\partial}{\partial y^{\prime}}\left[\frac{1}{\varepsilon_{3}}\left(\frac{\partial H_{y^{\prime}}}{\partial x^{\prime}}-\frac{\partial H_{x^{\prime}}}{\partial y^{\prime}}\right)\right]-i k_{0} \mu_{1} H_{x^{\prime}},
\end{aligned}
$$

a) Electronic mail: zye@iastato.edu

b) Electronic mail: kmh

$$
\begin{aligned}
& \frac{\partial}{\partial s} H_{x^{\prime}}=\frac{1}{i k_{0}} \frac{\partial}{\partial x^{\prime}}\left[\frac{1}{\mu_{3}}\left(\frac{\partial E_{y^{\prime}}}{\partial x^{\prime}}-\frac{\partial E_{x^{\prime}}}{\partial y^{\prime}}\right)\right]-i k_{0} E_{2} E_{y^{\prime}}, \\
& \frac{\partial}{\partial s} H_{y^{\prime}}=\frac{1}{i k_{0}} \frac{\partial}{\partial y^{\prime}}\left[\frac{1}{\mu_{3}}\left(\frac{\partial E_{y^{\prime}}}{\partial x^{\prime}}-\frac{\partial E_{x^{\prime}}}{\partial y^{\prime}}\right)\right]+i k_{0} \varepsilon_{1} E_{x^{\prime}} \cdot
\end{aligned}
$$

where

$$
\begin{aligned}
& \varepsilon_{1}=\varepsilon_{2}=\varepsilon \alpha, \\
& \mu_{1}=\mu_{2}=\mu \alpha, \\
& \varepsilon_{3}=\varepsilon / \alpha, \\
& \mu_{3}=\mu / \alpha, \\
& \alpha=\left(1-\frac{x^{\prime}}{R}\right) .
\end{aligned}
$$

So the arc structure can be viewed as a straight one with effective $\bar{\varepsilon}$ and $\bar{\mu}$ tensors depending on the transverse coordinate $x^{\prime}$ :

$$
\begin{aligned}
& \bar{\varepsilon}=\left[\begin{array}{ccc}
\varepsilon_{1} & 0 & 0 \\
0 & \varepsilon_{2} & 0 \\
0 & 0 & \varepsilon_{3}
\end{array}\right] . \\
& \tilde{\mu}=\left[\begin{array}{ccc}
\mu_{1} & 0 & 0 \\
0 & \mu_{2} & 0 \\
0 & 0 & \mu_{3}
\end{array}\right] .
\end{aligned}
$$

Equations (5)-(7) have almost the same form as in Sacks' uniaxial perfectly matched layer (UPML), i.e., no reflection occurs at a plane interface between two media with the same $(\varepsilon, \mu)$ but different $\alpha$ in Eqs. (5)-(7). ${ }^{11}$ The difference lies in that $\alpha$ of PML is a complex constant which can give an absorption. Here $\alpha$ is a real number and varies with $x^{\prime}$. The curved part of the waveguide is perfectly matched with the straight part in the $s$ direction, but not in the $x^{\prime}$ and $y^{\prime}$ directions. 


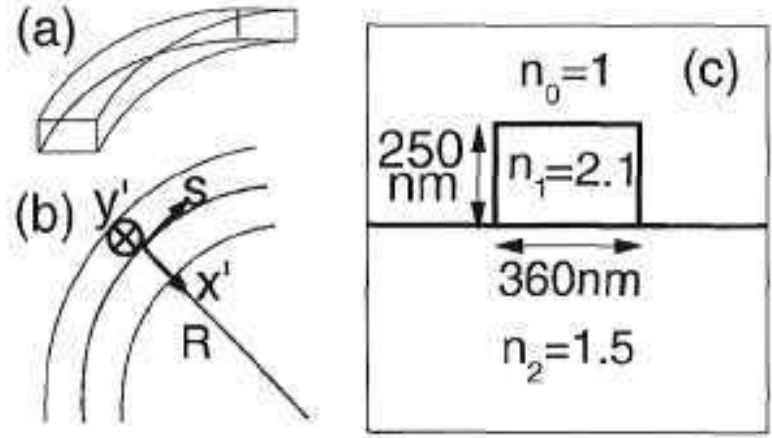

FIG. 1. Structure of an are nanoribbon waveguide on substrate. (a) 3D view, (b) top view with curvilinear coordinates $\left(x^{\prime}, y^{\prime}, s\right)$, and (c) eross-section illustration with size and material parameter set.

When the curved waveguide is effectively considered as a straight one with $(\tilde{\varepsilon}(x), \vec{\mu}(x))$ given by Eqs. $(5)-(7)$, we can apply the well-developed plane wave TMM (Refs. 7-9) to calculate the dispersion relations and eigenmode profiles in the curved waveguide in the same way as in a straight waveguide. ${ }^{12}$ In the following, we consider a nanoribbon with a cross section of $360 \mathrm{~nm}\left(x^{\prime}\right) \times 250 \mathrm{~nm}\left(y^{\prime}\right)$ on a substrate, where the refractive indices $n_{0}=1.0, n_{1}=2.1, n_{2}=1.5$ [see Fig. 1(c), these parameters are close to the ones in Ref. 1].

Figures 2(a) and 2(b) show respectively the dispersion relations for a straight waveguide and an are one with $R=2 \mu \mathrm{m}$; the insets give the $\left|E_{x}\right|\left(\left|E_{y}\right|\right)$ distribution of the first (second) eigenmode with $E_{x}\left(E_{y}\right)$ polarization at $600 \mathrm{~nm}$. The guided modes move downwards for a bent waveguide and the modal field shifts outwards from the center of curvature $\left(-x^{\prime}\right.$ direction). 13,14 The first and fourth modes are $E_{x}$ polarized, and the second and third modes are $E_{y}$ polarized. Modes after the fifth mode are not as highly polarized as the first four modes.

We consider the transmission coefficients for a curved waveguide joint between two straight waveguides. The transmission and reflection coefficients can be computed following the steps in Refs. 7-9. Here we only present the self-transmission coefficients of guided eigenmode $(i=1,2, \ldots)$, which are defined as the ratio of the transmission energy flux particular mode $i$ and the incident energy flux (only mode $i$ incident). The reason for doing this is that in propagation along the straight waveguide, we expect that some guided modes are much more sensitive to waveguide imperfections (such as sidewall roughness) than others. These sensitive modes are much more likely to disappear during propagation.

We start from a simple " $U$ " shape structure, made of two semi-infinite straight waveguides connected by a semicircular waveguide [see the inset in Fig. 3(a)]. First we set $R$ to be $10 \mu \mathrm{m}$. The self-transmission of the first six guided eigenmodes is shown in Fig. 3(a). One can see regular fluctuations in transmission like in Ref. 1 but with much weaker amplitude. Our calculation shows that the reflection is very small $\left(<10^{-4}\right.$, not shown here). That agrees well with the UPML form of $\bar{\varepsilon}$ and $\bar{\mu}$ in Eqs. (5)-(7), which indicates little reflection if the bend is not very sharp. Then we try a " $\mathrm{L}$ " structure, made of two semi-infinite straight waveguides connected by a quarter-circular waveguide with $R=20 \mu \mathrm{m}$. The result is shown in Fig. 3(b). The amplitude of transmission fluctuation is even weaker, but the position of the transmis-

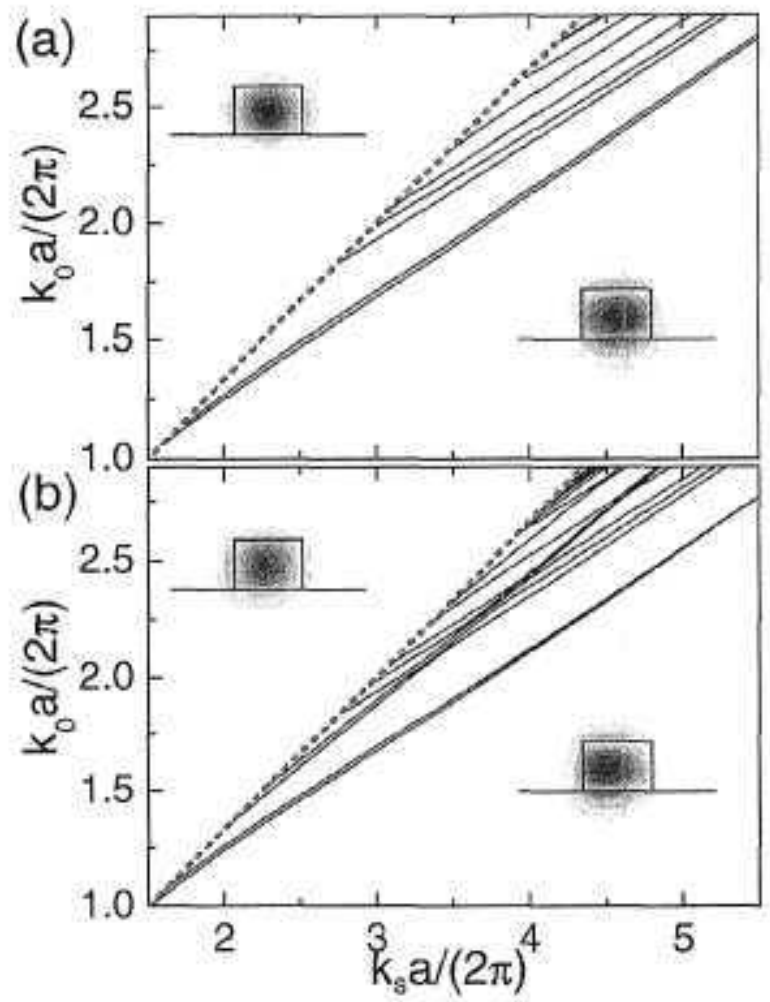

FIG. 2. (Color online) Dispersions of guided modes for (a) a straight waveguide and (b) a curved wsveguide with $R=2 \mu \mathrm{m}$. Here $a$ is the supercell constant and $a=1, \mu \mathrm{m}$. The dashed line is the light line in substrate: $k_{3}=n_{2} k_{0}\left(k_{0}=2 \pi / \lambda\right)$. The left (right) insets are $\left|E_{3}\right|\left(\left|E_{,}\right|\right)$distribution of the first (second) mode at $\lambda=600 \mathrm{~nm}$.

sion peaks and bottoms of the first four modes are about the same compared to Fig. 3(a). It is interesting that the first two $E_{x}$-polarized modes (the first and fourth modes) have almost the same period, and the first two $E_{\mathrm{y}}$-polarized modes (the second and third modes) also do so. We tested structures with different curvatures and different span lengths. We found that (i) the amplitude of transmission fluctuation decreases as $R$ is increased and (ii) the period of transmission fluctuation is only related to the span length of the arc part and decreases as we extend the are part. The first rule is natural to understand. Smaller radius of curvature enlarges the perturbation to the system, causing the transmission to fluctuate more intensively [see Fig. 3(c), where $R$ is set to be $4 \mu \mathrm{m}$ ]. The second rule can be explained by mode conversion. Let us begin from a simple model. Suppose there are two modes in the waveguide marked $i$ and $j$ for the straight part and $i^{\prime}$ and $j^{\prime}$ for the curved part. If the are part has length $L$, we can write down the self-transmission of mode $i$ when the reflection is very small:

$$
\begin{aligned}
& \left.\left|t_{i j^{\prime}} \exp \left(i k_{i}^{\prime} L\right) t_{j^{\prime} i^{\prime}}+t_{i j^{\prime}} \exp \left(i k_{j}^{\prime} L\right) t_{j}\right|^{2}\right|^{2}=\left|t_{i j^{\prime}}\right|^{4}+\left|t_{i^{\prime}}\right|^{4} \\
& \quad+2\left|t_{i j^{\prime}}\right|^{2}\left|t_{i j^{\prime}}\right|^{2} \cos \left(\Delta k^{\prime} L\right) .
\end{aligned}
$$

where $\Delta k^{\prime}=k_{j}^{\prime}-k_{i}^{\prime} ; t_{i i^{\prime}}, t_{i^{\prime}}, t_{i^{\prime}}, t_{j^{\prime}}$ are the conversion coefficients from mode $i$ to $i^{\prime}, i^{\prime}$ to $i, i$ to $j^{\prime}, j^{\prime}$ to $i$, respectively, and $t_{i i^{\prime}}=t_{i{ }^{\prime}}^{*}, t_{i j^{\prime}}=t_{j^{\prime} j^{*}}$. Because $\Delta k^{\prime}$ is not very sensitive to $R$, the period of transmission fluctuation is mainly related to the span length $L$ of the arc part. The conversion between modes with the same polarization is much stronger than that between different polarization modes. So the first and fourth 


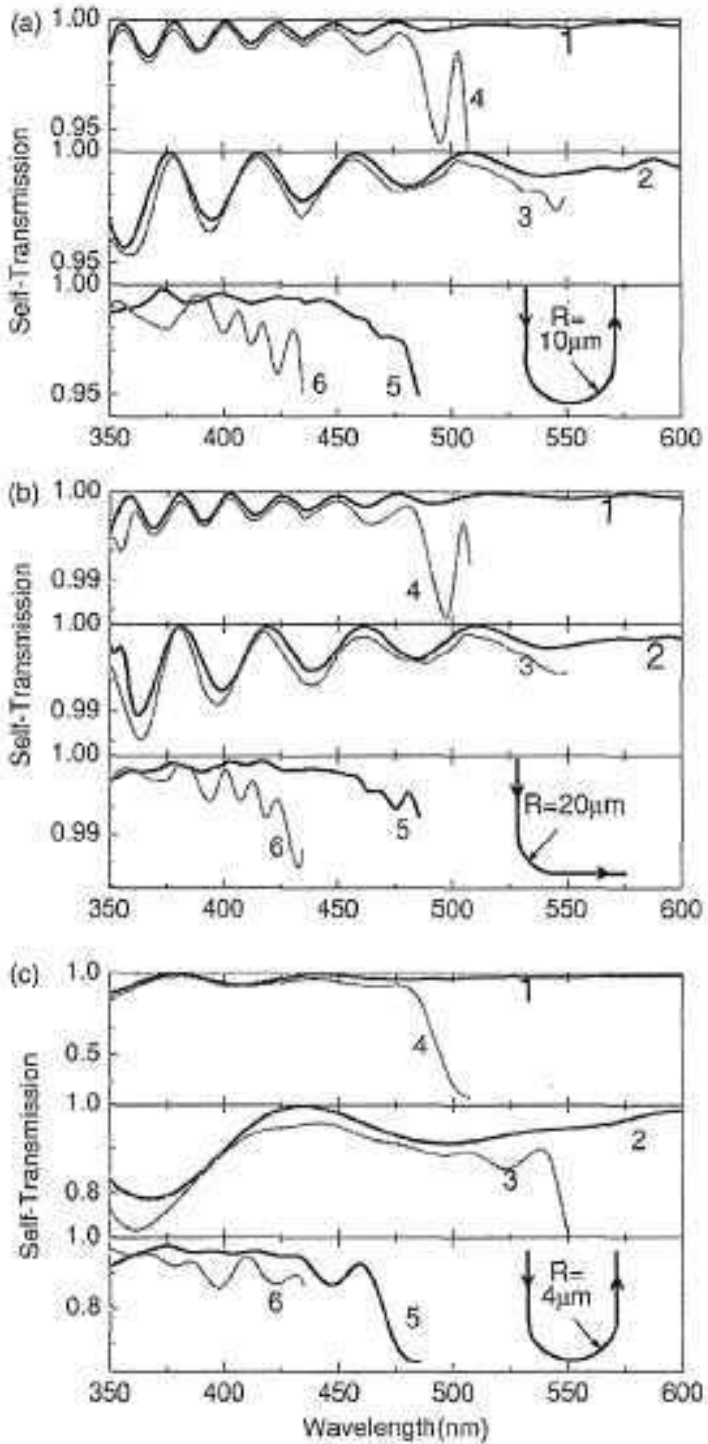

FIG. 3. Self-transmission of the first six modes for $\langle a\rangle$ a "U" structure of $R=10 \mu \mathrm{m}$; (b) an "L" structure of $R=20 \mu \mathrm{m}$, and (c) a "U" structure of $R=4 \mu \mathrm{m}$.

modes have similar self-transmission periods and the second and third modes also have similar periods. However, higher order modes are not highly polarized in either the $x$ or the $y$ direction. The conversion rates between different polarized modes are not small. So their self-transmissions do not show fluctuations as regular as the first four modes [see the bottom plots of Figs. 3(a) and 3(b)].

Our numerical results suggest a possible explanation for the strong regular oscillation in the output spectrum observed by Law et al. ${ }^{1}$ The observed oscillations can be caused by a rippling section of nanoribbon (as shown in the inset of Fig. 4), which has a small bending radius for the curved part.

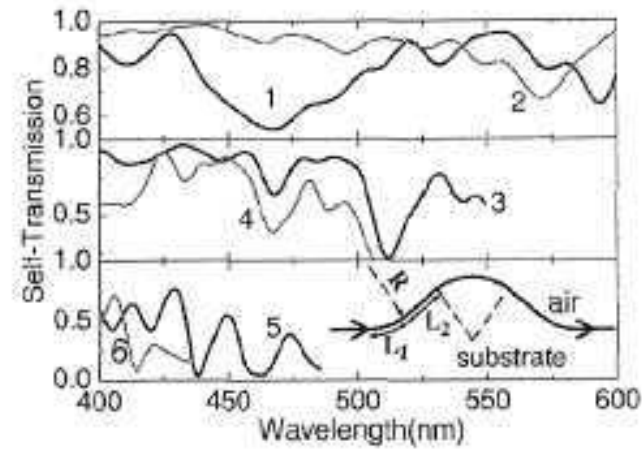

FIG. 4. Self-transmission of the first six modes for the structure in the inset bot of two periods of fluctuation, when $R=3 \mu \mathrm{m}, L_{1}=1 \mu \mathrm{m}$, and $L_{2}=5 \mu \mathrm{m}$. Inset. Illustration of a waveguide containing a rippling section.

We calculate the structure in the inset of Fig. 4 for different parameters, and one of the results is shown in Fig. 4. The parameters are $R=3 \mu \mathrm{m}, L_{1}=1 \mu \mathrm{m}, L_{2}=5 \mu \mathrm{m}$, and two periods of fluctuations (the inset of Fig. 4 only shows one period). The self-transmission diagram exhibits similar strong oscillations in the third, fourth, and fifth modes as in Ref. 1.

In summary, we have developed an improved TMM method in curvilinear coordinates to study curved nanoribbon waveguides. Our method can be applied to any shape of curved waveguides. From our results we can extract and explain two rules concerning the period and amplitude of the transmission fluctuations. We finish by calculating a rippling waveguide structure and obtain oscillations in transmission similar to those observed in experiments.'

This work was supported by Department of Energy under Project No. DE-AC-02-05CH11231. The Ames Laboratory is operated for the U.S. Department of Energy by Iowa State University under Contract No. W-7405-ENG-82.

M. Law, D. J. Sirbuly, J. C. Johnson, J, Goldberger, R. J. Saykally, and P Yang Science 305, 1269 (2004)

${ }^{2} \mathrm{C}$. J. Barrelet, A. B. Greytak, and C. M. Lieber, Nano Lett. 4, 1981 (2004).

M. H. Huang, S. Mao, H. Feick, H Q. Yan, Y. Y. Wu, H. Kind, E. Weber, R. Russo, and P. D. Yang, Science 292, 1897 (2001).

'X. Duan, Y. Huang, R. Agarwal, and C. M. Lieber, Natire (London) 421, $241(2003)$

${ }^{5}$ A. V. Masloy and C. Z. Ning, Appl. Pays. Lett. 83, 1237 (2003).

${ }^{6} \mathrm{H}$. Kind, H. Yan, B. Messer, M. Law, and P. Yang, Adv, Mater. (Weinheim, Ger.) 14, $158(2002)$.

'Z, Y. Li and L. L. Lin, Phys. Rev, E 67, 046607 (2003)

2. Y. Li and K. M. Ho, Phys. Rev. B 68, 155101 (2003).

${ }^{9}$ Z. Y. Li, L L L Lin, and K. M. Ho, Appl. Phys. Lett. 84, 4699 (2004).

${ }^{10} M$. Skorobogatiy, S. A. Jacobs, S. G. Johnson, and Y. Fink. Opt. Express 10. $1227(2002)$

1 'Z. S. Sacks, D. M. Kingsland, R. Lee, and J. F. Lee, IEEE Trans. Antennas Propag. 43, 1460 (1995)

12 Z Y. Li and K. M. Ho, Phys. Rev. B 71,045315 (2005)

${ }^{13}$ V. Van, P. P. Absil, J. V. Hryniewicz, and P.-T. Ho, J. Lightwave Techach. 19. 1734 (2001)

${ }^{11}$ Y. A. Vlasov and S. 1. McNab, Opt. Express 12, 1622 (2004) 

Acknowledgements: This work was supported by the Director, Office of Science, Office of Basic Energy Sciences, Material Sciences and Engineering Division, of the U.S. Department of Energy under Contract No. DE-AC02-05CH11231. We thank the National Center for Electron Microscopy for the use of their facilities. 
